
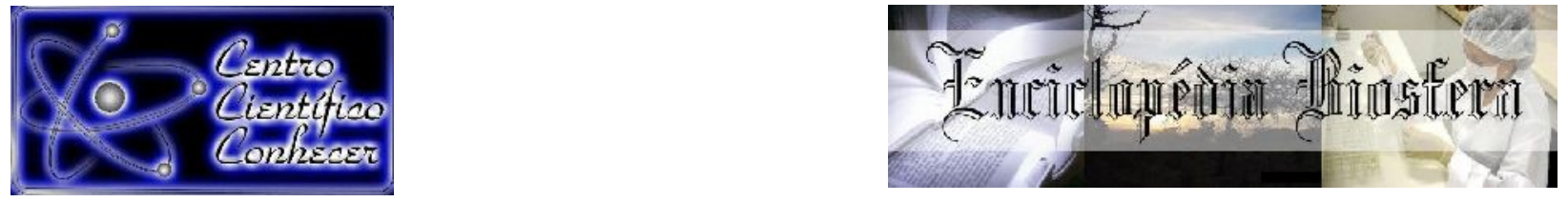

\title{
EPIDEMIOLOGIA DA TUBERCULOSE BOVINA NO MUNICÍPIO DE IBITIRAMA- ES EM 2018
}

\author{
Geneval da Motta Neto ${ }^{1}$, Eduardo Vargas de Oliveira ${ }^{2}$, Juliano Pereira Chaves ${ }^{3}$, \\ Dirlei Molinari Donatele ${ }^{4}$ \\ 1 Graduando em Medicina Veterinária da Universidade Federal do Espírito Santo \\ (UFES), Alegre-ES, Brasil. \\ E-mail: geneval-motta-neto@hotmail.com \\ ${ }^{2}$ Mestrando em Ciências Veterinárias da Universidade Federal do Espírito Santo \\ (UFES), Alegre-ES, Brasil. \\ ${ }^{3}$ Médico Veterinário do Instituto de Defesa Agropecuária e Florestal do Espírito \\ Santo (IDAF), Ibatiba-ES, Brasil. \\ ${ }^{4}$ Professor do curso de Medicina Veterinária da Universidade Federal do Espírito \\ Santo (UFES), Alegre-ES, Brasil.
}

Recebido em: 04/10/2019 - Aprovado em: 30/11/2019 - Publicado em: 15/12/2019 DOI: 10.18677/EnciBio_2019B26

\begin{abstract}
RESUMO
O objetivo do presente trabalho foi determinar a prevalência e os possíveis fatores de risco da Tuberculose Bovina no município de Ibitirama, sul do Espírito Santo. O estudo foi realizado em 48 propriedades do município, abrangendo um total de 901 bovinos. Os animais de cada propriedade foram testados para a Tuberculose Bovina (TB) utilizando-se primeiramente o teste cervical simples. Bovinos classificados como reagentes no primeiro teste foram submetidos ao teste cervical comparativo após 69 dias, quando solicitado a repetição pelo proprietário. Os testes foram realizados por um médico veterinário habilitado e todos os animais considerados positivos foram sacrificados. A prevalência de animais positivos para tuberculose bovina no município de lbitirama foi de 4,44\% [3,23 - 5,65], e a prevalência de focos de tuberculose foi de $39,58 \%$ [22,01 - 57,15]. O principal fator de risco para a TB encontrado neste estudo foi a aquisição de novos animais e, principalmente, quando estes não são testados para a doença antes de serem introduzidos na propriedade. O controle e erradicação da tuberculose bovina necessita de assistência veterinária de caráter preventivo, de forma que sejam feitos testes regularmente para identificar a doença na propriedade e sempre que houver a aquisição de novos animais.
\end{abstract}

PALAVRAS-CHAVE: Fator de risco. Mycobacterium bovis. Prevalência.

\section{EPIDEMIOLOGY OF BOVINE TUBERCULOSIS IN THE CITY OF IBITIRAMA-ES} IN 2018

\begin{abstract}
The objective of the present study was to determine the prevalence and possible risk factors of bovine tuberculosis in the municipality of Ibitirama, southern Espírito Santo. The study was conducted in 48 properties of the municipality, covering a total of 901 cattle. Animals from each property were tested for Bovine Tuberculosis using the simple cervical test first. Cattle classified as reagents in the first test were submitted
\end{abstract}


to a comparative cervical test after sixty to ninety days, when the repetition was requested. The tests were performed by a qualified veterinarian and all positive animals were sacrificed. The prevalence of positive animals of bovine tuberculosis in the city of Ibitirama was $4.44 \%$ [3.23 - 5.65] and the prevalence of outbreaks of tuberculosis was 39.58\% [22.01 - 57.15]. The main risk factor for TB (bovine tuberculosis) found in this study was the acquisition of new animals and especially when they are not tested for the disease before being introduced to the property. The control and eradication of bovine tuberculosis requires preventive veterinary assistance, so that tests are performed regularly to identify the disease on the property and whenever new animals are purchased.

KEYWORDS: Mycobacterium bovis. Prevalence. Risk factor.

\section{INTRODUÇÃO}

O município de Ibitirama possui um rebanho bovino total de 11.867 animais, dos quais, 2.859 são destinados a produção de leite (IBGE, 2019b). A pecuária leiteira é um sistema de produção amplamente utilizado no estado do Espírito Santo, pois gera renda mensal durante todo o ano para os produtores, favorecendo assim a permanência dos mesmos no campo (INCAPER, 2018).

A estrutura fundiária da região é constituída essencialmente de pequenas propriedades, de base familiar. O setor agropecuário representa a base econômica e social de lbitirama, sendo sua principal fonte geradora de emprego e renda. A principal atividade agrícola é o café, seguido da bovinocultura de leite (IBGE, 2019a).

A tuberculose bovina é uma doença infectocontagiosa de caráter zoonótico e sua importância econômica está relacionada principalmente com perdas diretas através da morte de animais, diminuição do ganho de peso, queda na produção de leite, descarte de animais e condenações de carcaças no momento do abate. Nos países em que a doença está presente, a maioria dos casos humanos de tuberculose devido ao Mycobacterium bovis ocorre em indivíduos jovens e resultam do consumo ou manuseio do leite de animais contaminados (AMENI et al., 2003; BRASIL, 2006).

No ano de 2017 foram registrados 4.488 casos de tuberculose bovina em todo território nacional, destes casos, 24 ocorreram no Espírito Santo (BRASIL, 2019). De acordo com estudos realizados por Davidson et al. (2017) no Reino Unido e Bapat et al. (2017) na Índia, a forma mais comum de exposição ao agente infeccioso para os humanos é o consumo de leite não pasteurizado. A principal fonte de infecção dos bovinos são outros bovinos acometidos pela tuberculose. O agente infeccioso é transmitido especialmente pela via aérea, a partir da inalação de aerossóis contaminados (DUMONT et al. 2018).

A tuberculose geralmente mostra uma evolução prolongada ao longo do tempo, e os sintomas podem levar meses ou anos para aparecer. Animais infectados podem apresentar fraqueza, perda de apetite com consequente perda de peso, febre intermitente, tosse seca, diarreia e aumento de linfonodos. Em alguns casos, no entanto, a bactéria pode permanecer latente no organismo hospedeiro sem desencadear a doença (OIE, 2019).

Santos e Alessi (2016) afirmam que em aproximadamente $10 \%$ dos bovinos com tuberculose pulmonar, ocorre extensão do processo infeccioso para a cavidade nasal, faringe e traqueia. Desse modo, são observados nódulos ou pequenos pólipos de tamanhos variados, firmes, coloração avermelhada, com material 
necrótico de aspecto purulento ou mineralizado em seu interior, acarretando dispneia e disfagia.

Ao longo do século passado, países que fizeram uso de programas de controle da tuberculose animal, com bases em tuberculinização e abate sanitário dos animais positivos, conseguiram reduzir consideravelmente o número de animais infectados (BRASIL, 2006).

De acordo com PNCEBT (Programa Nacional de Controle e Erradicação da Brucelose e da Tuberculose Animal) o diagnóstico alérgico-cutâneo com tuberculina é fundamental para programas de controle e erradicação da tuberculose bovina. Este tipo de diagnóstico pode identificar animais positivos, a partir de três a oito semanas da exposição ao Mycobacterium, alcançando boa sensibilidade e especificidade (BRASIL, 2017).

Apesar de diversos estudos sobre vacinação e tratamento da tuberculose bovina, os resultados obtidos não justificam a adoção dessas medidas como forma de controle da enfermidade. Além disso, em países que alcançaram grande sucesso com programas implementados para o combate à tuberculose bovina, essas práticas não foram utilizadas; e, portanto, não estão contempladas na estratégia de ação do PNCEBT (BRASIL, 2006).

Sendo assim, é importante que os produtores rurais mantenham a segurança e sanidade dos rebanhos, realizando monitoramento contínuo dos animais, além de aderir aos testes de tuberculina como parte do manejo da propriedade, descartando imediatamente os animais que apresentem positividade para o exame, afim de que não disseminem mais as bactérias pelas pastagens, possibilitando a infecção de outros animais (GERMANO; GERMANO, 2003).

O objetivo do presente trabalho foi determinar a prevalência da Tuberculose Bovina, e os principais fatores de risco da infecção em rebanhos bovinos no município de Ibitirama, sul do Espírito Santo.

\section{MATERIAL E MÉTODOS}

O presente estudo transversal, registrado sob o número 005/2018 e aprovado pela Comissão de Ética no Uso de Animais - Campus de Alegre (CEUA-ALEGRE) foi realizado em 48 propriedades de Ibitirama-ES abrangendo um total de 901 bovinos. Localizado na Serra do Caparaó, região sul do Espírito Santo, o município é predominantemente acidentado e montanhoso, com altitude média superior a 1500 metros. O Pico da Bandeira fica localizado em Ibitirama e se caracteriza como o ponto mais alto do Espírito Santo possuindo 2.891,32 metros de altitude (PMI, 2018).

As técnicas empregadas para o diagnóstico, estabelecidas pelo Manual Técnico do PNCEBT (Instrução Normativa DAS/MAPA no 10 do MAPA, de 3 de março de 2017), foram o teste cervical simples (TCS) e o teste cervical comparado (TCC). Os testes foram realizados durante o período de janeiro a dezembro de 2018, por um médico veterinário habilitado no PNCEBT, em serviço particular.

Os proprietários entraram em contato com o profissional, objetivando a avaliação da presença de tuberculose bovina em seus rebanhos, então, o mesmo encaminhou-se às propriedades requerentes e aplicou primeiramente o teste cervical simples, e após 69 dias o teste cervical comparado, quando solicitado a repetição pelo proprietário. O TCS e o TCC foram realizados segundo preconizado pela Instrução Normativa SDA/MAPA no 10 de 3 de março de 2017. Durante as visitas para realização das leituras e interpretação dos resultados, foi aplicado um questionário epidemiológico, visando identificar os fatores de risco para a presença de tuberculose bovina nas propriedades avaliadas. 
Em relação ao questionário empregado, buscou-se estudar as seguintes variáveis: tipo de exploração (corte, leite ou mista); tipo de criação (confinado, semiconfinado ou extensivo); número de ordenhas por dia (uma ordenha, 2 ou 3 ordenhas ou não ordenha); tipo de ordenha (mecânica ou manual); produção de leite diária em média, classificada de acordo com Barros et al. (2001), sendo que até $53 \mathrm{~L}$ por dia é considerado pequeno produtor de leite, de 53 a $133 \mathrm{~L}$ por dia é classificado como médio e acima de $133 \mathrm{~L}$ por dia como grande produtor de leite; presença de outras espécies na propriedade (ovinos/caprinos, equídeos, suínos, cão, gato); presença de espécies silvestres em vida livre na propriedade (não há presença, capivaras, marsupiais); se a pastagem faz divisa com mata; realização de testes para diagnóstico de tuberculose e com que regularidade; aquisição de bovinos nos últimos dois anos e se foram tuberculinizados; se aluga pasto em alguma época do ano; para quem entrega o leite (cooperativa, laticínio, direto ao consumidor ou não entrega); resfriamento do leite (não faz, tanque de expansão próprio ou coletivo); se produz queijo e/ou manteiga na propriedade e sua finalidade; consome leite cru (não pasteurizado), assistência veterinária (não possui, cooperativa/extensão ou particular); se compartilha aguadas ou bebedouros com outras propriedades e se realiza o período de quarentena após a aquisição de novos animais.

Para o cálculo da prevalência utilizou-se as seguintes equações matemáticas:

$$
\begin{gathered}
\text { Prevalência de animais positivos }=\frac{N 2 \text { de Casos Positivos }}{\text { Total de animais avaliados }} \times 100 \\
\text { Prevalência de focos de tuberculose }=\frac{N \text { de Propriedades Positivas }}{\text { Total de propriedades avaliadas }} \times 100
\end{gathered}
$$

No entanto, como estudou-se uma amostra e, não, todos os animais do município, essa medida não apresenta $100 \%$ de certeza, sendo necessária a correção dos valores obtidos, por meio do cálculo do Intervalo de Confiança com 95\% (IC 95\%), conforme equação:

$$
\text { IC } 95 \%=P \pm 1,96 \sqrt{(P(1-P) / N}
$$

Foram adotados como parâmetros para fins de cálculos os seguintes critérios: nível de confiança de 0,95 e erro de 0,05. As variáveis obtidas pelo questionário foram tabuladas e as associações entre as variáveis e a ocorrência de tuberculose foram realizadas no programa OpenEpi, com a realização do teste de associação do Qui-quadrado ou do Teste Exato Mid-P, e cálculo da medida de associação Odds Ratio.

\section{RESULTADOS E DISCUSSÃO}

A prevalência de animais positivos para tuberculose bovina no município de Ibitirama foi de 4,44\% [3,23 - 5,65], e a prevalência de focos de tuberculose foi de $39,58 \%$ [22,01 - 57,15]. Na Tabela 1 observa-se a prevalência total e de cada propriedade avaliada neste estudo. Se comparado aos resultados obtidos por Galvis et al., (2016) no estado do Espírito Santo, quando a prevalência de animais positivos foi de $0,7 \%[0,3$ - 1,1] e a prevalência de propriedades foi de 7,6\% [5,7 - 9,9], percebe-se que os valores evidenciados no presente estudo foram consideravelmente superiores. 
TABELA 1. Prevalência de tuberculose bovina em propriedades do município de Ibitirama, 2018.

\begin{tabular}{|c|c|c|c|}
\hline Propriedade & № Animais Avaliados & № Animais Positivos & Prevalência (\%) \\
\hline A & 25 & 1 & 4,00 \\
\hline B & 17 & - & - \\
\hline C & 10 & - & - \\
\hline $\mathrm{D}$ & 12 & - & - \\
\hline$E$ & 10 & 3 & 30,00 \\
\hline $\mathrm{F}$ & 13 & - & - \\
\hline$G$ & 17 & - & - \\
\hline $\mathrm{H}$ & 8 & - & - \\
\hline 1 & 7 & - & - \\
\hline J & 6 & 1 & 16,67 \\
\hline $\mathrm{K}$ & 10 & 2 & 20,00 \\
\hline $\mathrm{L}$ & 23 & - & - \\
\hline$M$ & 23 & 2 & 8,70 \\
\hline $\mathrm{N}$ & 3 & 1 & 33,33 \\
\hline O & 21 & - & - \\
\hline $\mathrm{P}$ & 13 & - & - \\
\hline Q & 18 & - & - \\
\hline $\mathrm{R}$ & 33 & 1 & 3,03 \\
\hline$S$ & 28 & 1 & 3,57 \\
\hline $\mathrm{T}$ & 10 & - & - \\
\hline$U$ & 7 & - & - \\
\hline $\mathrm{V}$ & 10 & - & - \\
\hline W & 18 & 2 & 11,11 \\
\hline$x$ & 14 & 1 & 7,14 \\
\hline$Y$ & 17 & 1 & 5,88 \\
\hline Z & 28 & - & - \\
\hline$A A$ & 13 & - & - \\
\hline BB & 53 & 1 & 1,89 \\
\hline $\mathrm{CC}$ & 16 & - & - \\
\hline DD & 10 & - & - \\
\hline EE & 129 & 6 & 4,65 \\
\hline $\mathrm{FF}$ & 14 & 6 & 42,86 \\
\hline GG & 25 & 1 & 4,00 \\
\hline $\mathrm{HH}$ & 30 & 4 & 13,33 \\
\hline II & 10 & - & - \\
\hline $\mathrm{JJ}$ & 11 & - & - \\
\hline KK & 14 & - & - \\
\hline $\mathrm{LL}$ & 15 & - & - \\
\hline MM & 8 & - & - \\
\hline $\mathrm{NN}$ & 15 & - & - \\
\hline $\mathrm{OO}$ & 8 & - & - \\
\hline PP & 2 & - & - \\
\hline
\end{tabular}




\begin{tabular}{cccc}
\hline Propriedade & № Animais Avaliados & № Animais Positivos & Prevalência (\%) \\
\hline QQ & 18 & - & - \\
RR & 17 & - & - \\
SS & 36 & 1 & 2,78 \\
TT & 9 & - & - \\
UU & 17 & - & - \\
VV & 30 & 5 & 16,67 \\
\hline Total & $\mathbf{9 0 1}$ & $\mathbf{4 0}$ & $\mathbf{4 , 4 4}$ \\
\hline
\end{tabular}

$\mathrm{N}=48$ Propriedades.

Estados que fazem divisa com o Espírito Santo, como Bahia e Minas Gerais, realizaram estudos semelhantes nos últimos anos. Os valores de prevalência de animais e prevalência de focos de tuberculose bovina encontrados por Barbieri et al. (2016) em Minas Gerais foram respectivamente 0,56\% [0,46 - 0,66] e 4,25\% [3,36 $5,15]$. E no estado da Bahia, Bahiense et al. (2016) encontraram valores de 0,21\% [0,07 - 0,60] para a prevalência de animais e 1,6\% [1,0 - 2,6] para focos de tuberculose.

A elevada prevalência de animais e de focos no município de Ibitirama em relação ao estado do Espírito Santo, e outros estados brasileiros, evidencia que a doença se encontra disseminada na localidade, sendo que a cada 10 propriedades avaliadas, aproximadamente quatro apresentaram pelo menos um caso de tuberculose bovina, demostrando a necessidade de ações imediatas e efetivas dos órgãos de defesa sanitária animal e humana para o controle e prevenção da doença no município e regiões vizinhas. A Tabela 2 demonstra os possíveis fatores de risco encontrados para a disseminação da tuberculose bovina no município de lbitiramaES.

TABELA 2. Resultados obtidos para os possíveis fatores de risco para tuberculose bovina no município de lbitirama-ES, 2018.

\begin{tabular}{lccc}
\hline \multicolumn{1}{c}{ Variável } & Expostos/Casos & Expostos/Controle & $\boldsymbol{p}$ \\
\hline Tipo de Exploração (Leite) & $33 / 686$ & $7 / 215$ & $0,3342^{*}$ \\
Tipo de Criação (Confinamento) & $1 / 26$ & $39 / 875$ & $0,9793^{* *}$ \\
No Ordenhas (2 ou 3) & $34 / 770$ & $6 / 131$ & $0,9326^{*}$ \\
Tipo de Ordenha (Manual) & $16 / 221$ & $24 / 680$ & $0,01999^{*}$ \\
Produção de Leite (Até 53 L/dia) & $1 / 5$ & $39 / 896$ & $0,2212^{* *}$ \\
Produção de Leite (53 até 133 L/dia) & $8 / 213$ & $32 / 688$ & $0,5793^{*}$ \\
Produção de Leite (Acima de 133 L/dia) & $19 / 548$ & $21 / 353$ & $0,07748^{*}$ \\
Presença de Equídeos & $2 / 156$ & $38 / 745$ & $0,03524^{*}$ \\
Presença de Suínos & $8 / 257$ & $32 / 644$ & $0,2224^{*}$ \\
Presença de Cães ou Gatos & $14 / 305$ & $26 / 596$ & $0,8752^{*}$ \\
Presença de Marsupiais & $30 / 550$ & $10 / 351$ & $0,06408^{*}$ \\
Pastos com Divisa com Matas & $17 / 359$ & $23 / 542$ & $0,7257^{*}$ \\
Faz Testes de Tuberculose & $25 / 620$ & $15 / 281$ & $0,3780^{*}$ \\
Aquisição de Bovinos & $35 / 650$ & $5 / 251$ & $0,02666^{*}$ \\
nos Últtimos 2 Anos & & & \\
Aquisição de Bovinos & $25 / 282$ & $15 / 619$ & $0,00001341^{*}$ \\
Sem Tuberculinização & & & \\
Aluga Pastos & $8 / 193$ & $32 / 708$ & $0,8227^{*}$ \\
Compartilha Bebedouros/Aguadas & $3 / 170$ & $37 / 731$ & $0,06014^{*}$ \\
com outras propriedades & & & \\
\hline *Qui-quadrado Sem Correção; **Teste Exato Mid-P & &
\end{tabular}

${ }^{*}$ Qui-quadrado Sem Correção; **Teste Exato Mid-P 
As variáveis: assistência veterinária (não possui, cooperativa/extensão ou particular); e realiza período de quarentena após a aquisição de novos animais, não foram calculadas, pois $100 \%$ das propriedades deste estudo relataram que possuíam assistência veterinária particular e nenhuma realizava período de quarentena.

Após a análise dos possíveis fatores de risco para tuberculose bovina, as seguintes variáveis obtiveram associação significativa à doença: ordenha manual ( $p$ $=0,01999)$, presença de equídeos $(p=0,03524)$, aquisição de bovinos nos últimos dois anos $(p=0,02666)$ e aquisição de bovinos sem tuberculinização ( $p=$ 0,00001341 ). As demais variáveis não obtiveram associação com a tuberculose bovina, pois apresentaram valor de $p>0,05$.

Não houve associação entre criação de bovinos leiteiros com a tuberculose bovina nesta pesquisa, porém Veloso et al. (2016) concluíram em estudo no estado de Santa Catarina que rebanhos leiteiros apresentam maior risco de adquirir a enfermidade. Este resultado pode estar relacionado ao fato de a tuberculose bovina possuir uma evolução crônica, e por este motivo, ser comumente diagnosticada em rebanhos leiteiros, onde os animais permanecem na propriedade por maior período de tempo.

Apesar da variável № ordenhas (2 ou 3) não ter apresentado significância estatística no município de Ibitirama-ES, Rocha et al. (2016) encontraram associação da variável com a presença da tuberculose bovina no estado de Goiás. Propriedades que realizavam duas ou três ordenhas por dia obtiveram maior probabilidade de adquirir a tuberculose bovina do que propriedades que realizavam uma ordenha ou que não ordenhavam seus animais.

A medida de associação, usando a função Odds Ratio (OR), demonstrou que as variáveis: ordenha manual, aquisição de bovinos nos últimos dois anos e aquisição de bovinos sem tuberculinização são fatores causais para a ocorrência de tuberculose bovina no município de Ibitirama-ES (Tabela 3).

TABELA 3. Medida de associação da análise das variáveis estudadas com a ocorrência de tuberculose bovina no município de Ibitirama/ES, 2018.

\begin{tabular}{lccc}
\multicolumn{1}{c}{ Variável } & $\boldsymbol{p}$ & Odds Ratio & IC 95\% \\
\hline Tipo de Ordenha (Manual) & $0,01999^{*}$ & 2,133 & $1,112-4,093$ \\
Presença de Equídeos & $0,03524^{*}$ & 0,2416 & $0,05768-1,012$ \\
Aquisição de Animais nos Últimos 2 Anos & $0,02666^{*}$ & 2,8 & $1,084-7,23$ \\
Aquisição de Animais Sem Tuberculinização & $0,00001341^{*}$ & 3,917 & $2,032-7,552$ \\
\hline
\end{tabular}

No presente estudo, bovinos que eram ordenhados manualmente apresentaram um risco maior para a TB, do que os que eram ordenhados mecanicamente, resultado este, diferente do encontrado por Belchior et al. (2016), em que propriedades com algum grau de tecnificação obtiveram maior prevalência da doença. De acordo com Rosa et al. (2009), a introdução da tecnologia na extração do leite possibilitou uma ordenha mais rápida e sendo assim, os animais ficariam menos tempo em contato com os outros em currais ou salas de ordenha, local em que o risco de transmissão da tuberculose é maior. Diferente de Galvis et al. (2016), que identificaram a ordenha mecânica como um fator de risco, pois como as salas de ordenha possuem maior e melhor infraestrutura, são mais propensas a deterem maior densidade de animais.

A presença de equídeos, de alguma maneira, está relacionada à TB. Ainda não se conhece se esta variável se apresenta como um fator de risco ou um fator 
protetor, pois o Odds Ratio variou de 0,05768 a 1,012. Estudos semelhantes a este, como o de Vendrame e colaboradores (2016) no estado de Rondônia, não obtiveram associação entre esta variável e a enfermidade, levando a necessidade de mais estudos sobre essa associação.

O principal fator de risco para a TB encontrado neste estudo foi a aquisição de novos animais e, especialmente, quando estes não são testados para a doença antes de serem introduzidos na propriedade. Resultado semelhante obtido por Vendrame et al. (2016) no estado de Rondônia. De acordo com Abrahão (1999), a tuberculose bovina é introduzida em um rebanho, principalmente, pela aquisição de animais infectados, podendo se propagar nos bovinos, independentemente da idade, sexo e raça.

Segundo O'Hagan et al. (2016) os fatores de risco associados a tuberculose bovina podem ser potencialmente reduzidos, adotando-se medidas de biossegurança, como a realização de testes antes da introdução de novos animais no plantel.

Apesar de todas as propriedades avaliadas neste estudo possuírem algum tipo de assistência veterinária, não foi possível afirmar se a mesma é somente para emergências ou para prevenção de doenças. $O$ controle e erradicação da tuberculose bovina necessita de assistência veterinária de caráter preventivo, de forma que sejam feitos testes regularmente para identificar a doença na propriedade e sempre que houver a aquisição de novos animais.

É notório, que também a não realização do período de quarentena por todas as propriedades avaliadas é um motivo de preocupação para o elevado número de focos da doença no município.

\section{CONCLUSÕES}

O principal fator de risco para a propagação da tuberculose bovina no município de Ibitirama-ES é a aquisição de bovinos sem a realização do teste de tuberculinização. A prevalência tanto de animais, como de focos da doença foram consideradas alarmantes se comparadas aos valores obtidos no estado do Espirito Santo e outros estados brasileiros. Isto se deve, provavelmente, à introdução de novos animais nas propriedades do município sem qualquer tipo de controle sanitário.

\section{REFERÊNCIAS}

ABRAHÃO, REGINA MAURA CABRAL DE MELO. Tuberculose Humana Causada Pelo Mycobacterium bovis: Considerações Gerais e a Importâncias Dos Reservatórios Animais. Archives Of Veterinary Science, São Paulo, v. 4, n. 1, p.515, 1999. Disponível em: <https://revistas.ufpr.br/veterinary/article/view/3771>. DOI: 10.5380/avs.v4i1.3771.

AMENI, G.; AMENU, K.; TIBBO, M. Bovine Tuberculosis: Prevalence and Risk Factor Assessment in Cattle and Cattle Owners in Wuchale-Jida District, Central Ethiopia. The International Journal of Applied Research, Addis Ababa, v. 1, p.1-4, mar. 2003. Disponível em: <https://hdl.handle.net/10568/32984>.

BAHIENSE, L.; ÁVILA, L. N.; BAVIA, M. E.; AMAKU, M.; DIAS, R. A. et al. Prevalence and risk factors for bovine tuberculosis in the State of Bahia, Brazil. Semina: Ciências Agrárias, v. 37, n. 5, p. 3549-3559, 2016. Disponível em: 
<http://dx.doi.org/10.5433/1679-0359.2016v37n5Supl2p3549>. DOI: 10.5433/16790359.2016v37n5Supl2p3549.

BAPAT, P. R.; DODKEY, R. S.; SHEKHAWAT, S.; HUSAIN, A. A.; NAYAK, A. R. et al. Prevalence of zoonotic tuberculosis and associated risk factors in Central Indian populations. Journal of epidemiology and global health, v. 7, n. 4, p. 277-283, 2017. Disponível em: <https://www.ncbi.nlm.nih.gov/pubmed/29110869>. DOI: 10.1016/j.jegh.2017.08.007.

BARBIERI, J. M.; OLIVEIRA, L. F.; DORNELES, E. M. S.; MOTA, A. L.; GONÇALVES, V. S. et al. Epidemiological status of bovine tuberculosis in the state of Minas Gerais, Brazil, 2013. Semina: Ciencias Agrarias, 2016. Disponível em: <http://www.uel.br/revistas/uel/index.php/semagrarias/article/view/27405>. DOI: 10.5433/1679-0359.2016v37n5Supl2p3531.

BARROS, G. S. de C.; GALAN, V. B.; GUIMARÃES, V. Di A.; BACCHI, M. R. P. Sistema agroindustrial do leite no Brasil. Brasília, DF: Embrapa, 2001. 170 p.

BELCHIOR, A. P. C.; LOPES, L. B.; GONÇALVES, V. S. P.; LEITE, R. C. Prevalence and risk factors for bovine tuberculosis in Minas Gerais State, Brazil. Tropical Animal Health and Production, Edinburg, v. 48, n.2, p. 373-78, 2016.

BRASIL. Instrução Normativa SDA no 10, de 3 de março de 2017. Regulamento Técnico do Programa Nacional de Controle e Erradicação da Brucelose e da Tuberculose Animal - PNCEBT. Brasília, 2017.

BRASIL. Ministério da Agricultura, Pecuária e Abastecimento. Coordenação de Informação e Epidemiologia - Saúde Animal. Disponível em: $<$ http://indicadores.agricultura.gov.br/saudeanimal/index.htm>. Acesso em: 24 de abril de 2019.

BRASIL. Ministério da Agricultura, Pecuária e Abastecimento. Programa Nacional de Controle e Erradicação da Brucelose e da Tuberculose Animal (PNCEBT): Manual Técnico. Brasília, 2006. 188 p.

DAVIDSON, J. A.; LOUTET, M. G.; O'CONNOR, C.; KEARNS, C.; SMITH, R. M. et al. Epidemiology of Mycobacterium bovis disease in humans in England, Wales, and Northern Ireland, 2002-2014. Emerging infectious diseases, v. 23, n. 3, p. 377, 2017. Disponível em: <https://www.ncbi.nlm.nih.gov/pubmed/28220748>. DOI: 10.3201/eid2303.161408.

DUMONT, Y.; LOUNNAS, M.; CARRIÈRE, C.; SOLASSOL, J.; BAÑULS, A. et al. Tuberculose bovine: une maladie globalisée à l'interface homme-animal. Revue Francophone des Laboratoires, v. 2018, n. 503, p. 58-63, 2018. Disponível em: <https://doi.org/10.1016/S1773-035X(18)30179-5>. DOI: $10.1016 / \mathrm{S} 1773-$ 035X(18)30179-5.

GALVIS, J. O. A.; GRISI-FILHO, J. H.; COSTA, D.; SAID, L. P.; AMAKU, M. et al. Epidemiologic characterization of bovine tuberculosis in the state of Espírito Santo, Brazil. Semina: Ciências Agrárias, v. 37, n. 5, p. 3567-3578, 2016. Disponível em: 
<http://www.uel.br/revistas/uel/index.php/semagrarias/article/view/27262>.

DOI: 10.5433/1679-0359.2016v37n5Supl2p3567.

GERMANO, P.M.L.; GERMANO, M.I.S. Higiene e Vigilância Sanitária dos Alimentos. 2.ed. São Paulo: Livraria Varela, 2003.

IBGE. Instituto Brasileiro de Geografia e Estatística. Censo Agropecuário Resultados preliminares. Disponível em: <https://cidades.ibge.gov.br/brasil/es/ibitirama/pesquisa/24/76693> Acesso em: 20 de setembro de 2019a.

IBGE. Instituto Brasileiro de Geografia e Estatística. Produção da Pecuária Municipal. Disponível em: <https://cidades.ibge.gov.br/brasil/es/ibitirama/pesquisa/18/16459> Acesso em: 07 de setembro de 2019b.

INCAPER. Instituto Capixaba de Pesquisa, Extensão Rural e Assistência Técnica, 2018. Disponível em: <https://incaper.es.gov.br/pecuaria>. Acesso em: 12 de setembro de 2018.

O'HAGAN, M. J.; MATTHEWS, D. I.; LAIRD, C.; MCDOWELL, S. W. Herd-level risk factors for bovine tuberculosis and adoption of related biosecurity measures in Northern Ireland: a case-control study. The Veterinary Journal, v. 213, p. 26-32, 2016. Disponível em: <https://www.ncbi.nlm.nih.gov/pubmed/27240911>. DOI: 10.1016/j.tvjl.2016.03.021.

OIE. World Organisation for Animal Health. Bovine Tuberculosis. Disponível em: $<$ http://www.oie.int/en/animal-health-in-the-world/animal-diseases/bovinetuberculosis/>. Acesso em: 24 de abril de 2019.

PMI. Prefeitura Municipal de lbitirama, 2018. Disponível em: <http://ibitirama.es.gov.br/paginas.php?p=1>. Acesso em: 12 de setembro de 2018.

ROCHA, W. V.; JAYME, V. S.; MOTA, A. L.; BRITO, W. M.; PIRES, G. R. et al. Prevalence and herd-level risk factors of bovine tuberculosis in the State of Goiás, Brazil. Semina: Ciências Agrárias, v. 37, n. 5, p. 3625-3638, 2016. Disponível em: <http://www.uel.br/revistas/uel/index.php/semagrarias/article/view/27278>. DOI: 10.5433/1679-0359.2016v37n5Supl2p3625.

ROSA, M. S.; COSTA, M. J. R.; SANT'ANNA, A. C.; MADUREIRA, A. P. Manual de Boas Práticas de Manejo na Ordenha. Editora Funep: Jaboticabal-SP, 2009.

SANTOS, R. L.; ALESSI, A. C. Patologia Veterinária. 2. ed. Rio de Janeiro - RJ: Editora Roca, 2016. 1346 p.

VELOSO, F. P.; BAUMGERTEN, K. D.; MOTA, A. L.; FERREIRA, F.; NETO, J. S. et al. Prevalence and herd-level risk factors of bovine tuberculosis in the State of Santa Catarina. Semina: Ciências Agrárias, v. 37, n. 5, p. 3659-3672, 2016. Disponível em: <http://www.uel.br/revistas/uel/index.php/semagrarias/article/view/27282>. DOI: 10.5433/1679-0359.2016v37n5Supl2p3659. 
VENDRAME, F. B.; AMAKU, M.; FERREIRA, F.; TELES, E. O.; GRISI-FILHO, J. H. et al. Epidemiologic characterization of bovine tuberculosis in the State of Rondônia, Brazil. Semina: Ciências Agrárias, v. 37, n. 5, p. 3639-3646, 2016. Disponível em: <http://www.uel.br/revistas/uel/index.php/semagrarias/article/view/27280>. DOI: 10.5433/1679-0359.2016v37n5Supl2p3639. 